\title{
Impactos antropogénicos en la calidad del agua del río Cunas
}

\author{
Anthropogenic impacts in water quality of Cunas river
}

María Custodio Villanueva', Rafael Pantoja Esquivel ${ }^{2}$

Universidad Nacional del Centro del Perú

\section{RESUMEN}

Objetivos: Identificar los impactos que generan las actividades antropogénicas en la calidad del agua del río Cunas, en las provincias de Chupaca y Concepción del departamento de Junín en el año 2012. Métodos: Se utilizaron los métodos de observación, descripción y explicación; el tipo de investigación es básica, y el diseño no experimental, longitudinal. Se definieron tres estaciones de muestreo; la estación 1, se localizó en Angasmayo; la 2, en Antacusi; y la 3, en La Perla, Chupaca. Las muestras de agua fueron colectadas en botellas de plástico de dos litros y en frascos de vidrio estériles para determinar la concentración de nitratos y fosfatos, y coliformes termotolerantes, respectivamente. Los parámetros medidos in situ fueron: oxígeno disuelto $(\mathrm{mg} / \mathrm{l})$, sólidos totales disueltos ( $\mathrm{mg} / \mathrm{l})$, conductividad (mS/ $\mathrm{cm})$, temperatura $\left({ }^{\circ} \mathrm{C}\right), \mathrm{pH}$ y turbidez (FTU). Estas mediciones se realizaron con equipos portátiles. La identificación y valoración cualitativa de impactos se realizó previa identificación de las acciones de las actividades antropogénicas que se desarrollan en el río Cunas. Resultados: Los resultados de la calidad del agua fueron: estación 1 (Angasmayo), calidad media $(65,83)$; estación 2 (Antacusi), calidad media $(61,08) ;$ y estación 3 (La Perla), calidad media $(57,18)$. Conclusiones: En la estación 1 el impacto fue ligeramente moderado; en la 2, moderado; y en la 3 , severo.

Palabras clave: Río Cunas, impacto antropogénico, calidad de agua.

1 Bióloga, M. Sc. en Biotecnología. Docente investigadora de la Universidad Nacional del Centro del Perú.

2 Ingeniero Químico, Mg. en Educación Superior e Investigación. Docente investigador de la Universidad Nacional del Centro del Perú 


\section{ABSTRACT}

Objectives: Identify the impacts that generate the anthropogenic activities in the water quality of Cunas river, in Chupaca and Concepción provinces during 2012. Methods: The observational, descriptive and explanation methods were used, the research type is basic and the design is non experimental, longitudinal type. Itwas defined three sampling sites. Station 1 was located in Angasmayo, the station 2 in Antacusi and the station 3 in La Perla, Chupaca. Water samples were collected in plastic bottles of two liters and in sterile glass flasks to determine the concentration of nitrates and phosphates, and thermo-tolerant coliform bacteria, respectively. Parameters measured in situ were: dissolved oxygen $(\mathrm{mg} / \mathrm{l})$, total dissolved solids $(\mathrm{mg} / \mathrm{l})$, conductivity $(\mathrm{mS} /$ $\mathrm{cm})$, temperature $\left({ }^{\circ} \mathrm{C}\right), \mathrm{pH}$ and turbidity (FTU). These mensurations were carried out with portable instruments. The identification and qualitative valuation of impacts were carried out previous identification of the actions of the anthropogenic activities that are developed in the Cunas river. Results: Results of the water quality were: station 1 (Angasmayo), medium quality $(65,83)$; station 2 (Antacusi), medium quality $(61,08)$ and station 3 (La Perla), medium quality $(57,18)$. Conclusions: In the station 1 the impact was lightly moderate, in the station 2 was moderate and in the station 3 was severe.

Keywords: Anthropogenic impact, water quality, Cunas river.

\section{INTRODUCCIÓN}

El agua, además de ser un recurso imprescindible para la supervivencia del ser humano y el desarrollo de todas las formas de vida, es ampliamente utilizada en actividades diarias, como la agricultura (de $70 \%$ a $80 \%$ ), la industria (20\%), el uso doméstico (6\%), entre otras, convirtiéndose en uno de los recursos más apreciados en el planeta. Actualmente, la escasa disponibilidad de este recurso es motivo de preocupación no sólo para los expertos científicos, especialistas en la materia, - gobernantes, sino para la humanidad entera, que ha reconocido y comprendido la importancia que este recurso tiene para garantizar la vida del planeta.

No obstante que las fuentes de agua dulce representan un porcentaje mínimo de la disponibilidad de agua del planeta, muchos de los ríos en el Perú tienen el agravante problema de contaminación, debido al acelerado crecimiento desordenado de las comunidades humanas y de su desarrollo social, productivo y tecnológico. Por lo tanto, el desarrollo de las actividades humanas sin criterios ambientales, está afectando la salud del hombre y el estado de los sistemas acuáticos. En algunos casos provocando alteraciones de carácter irreversible.

En el contexto de los acuerdos ambientales globales, el tema del agua resulta preponderante no sólo por su carácter estratégico sino por la preocupación que existe en torno al problema de su escasez y calidad, más aún cuando este problema es causado por factores antropogénicos, los mismos que podemos evitar. De ahí que la calidad de agua potable, en especial, es un tema de preocupación y atención prioritaria para la Organización Mundial de la Salud porque el agua contaminada es un factor de múltiples enfermedades en la población. En tal sentido, el problema de investigación formulado fue: ¿ $Q$ ué impactos generan las actividades antropogénicas en la calidad del agua del río Cunas? Considerándose, como objetivo: Identificar los impactos que generan las actividades antropogénicas en la calidad del agua del río Cunas, en las provincias de Chupaca y Concepción en el 2012. El fin es ayudar a prevenir, evitar y corregir los efectos negativos del desarrollo de dichas actividades, procurando que se alcancen un aprovechamiento razonable de este recurso hídrico y se fortalezcan los efectos positivos.

La hipótesis planteada fue: Los impactos que generan la acuicultura, agricultura, 
ganadería, descargas de residuos líquidos domiciliarios y de residuos sólidos, están degradando la calidad del agua del río Cunas.

\section{MATERIAL Y MÉTODOS}

\section{Descripción del área de estudio}

La cuenca del río Cunas se ubica en la sierra central del Perú, sobre la margen derecha del río Mantaro, y tiene una extensión de 1845 km2; políticamente comprende las provincias de Chupaca y Concepción en la región Junín, complementado por una parte del distrito de Tomas en la provincia de Yauyos de la región Lima. Geográficamente, se encuentra ubicada entre las coordenadas $11^{\circ}$ $45^{\prime}$ y 12 을 $20^{\prime}$ latitud sur; 75으 15' y 75 $45^{\prime}$ ' longitud oeste.

\section{Descripción de las zonas de muestreo}

Fueron definidas tres estaciones de muestreo. La estación 1 se localizó a 10 metros del puente de Angasmayo; la 2, a 10 metros del puente de Antacusi; en tanto que la 3, a 10 metros del puente de La Perla, Chupaca.

La primera estación es una zona en la que predomina la actividad agrícola, seguida por la actividad piscícola. En esta zona existen viviendas que se asientan principalmente en la margen derecha del río Cunas, ejerciendo cierta presión sobre éste.

En el tramo de la estación 2 se han observado residuos sólidos y descarga de afluentes domésticos y de afluentes de explotaciones ganaderas. En la estación 3, en cambio, se observa residuos sólidos, lavado de zanahoria, lavado de ropa, descarga del afluente del centro de beneficio de animales, etc. La vegetación rivereña es escasa.

\section{Muestreo}

La recolección de las muestras de agua se realizó en junio de 2012 . Fueron tomadas dos muestras por estación. Una de las muestras fue para medir los parámetros físico-químicos y la otra para determinar coliformes termotolerantes.

\section{Análisis físico-químico del agua}

Las muestras de agua fueron colectadas en la superficie del río, en botellas de plástico de dos litros, y posteriormente llevadas al Laboratorio de Investigación de la Universidad Nacional del Centro del Perú, para el análisis de nitratos y fosfatos.

Los parámetros medidos in situ fueron: oxígeno disuelto $(\mathrm{mg} / \mathrm{l})$, sólidos totales disueltos $(\mathrm{mg} / \mathrm{l})$, conductividad $(\mathrm{mS} / \mathrm{cm})$, temperatura $\left({ }^{\circ} \mathrm{C}\right), \mathrm{pH}$ y turbidez (FTU). Para estas mediciones usamos equipos portátiles Hanna Instruments, en tanto que para los análisis de nitratos (mg/l) y fosfatos $(\mathrm{mg} / \mathrm{l})$, el fotómetro PC-MultiDirect.

\section{Análisis bacteriológico del agua}

Las muestras fueron tomadas en frascos de vidrio estériles y se refrigeraron a $4^{\circ} \mathrm{C}$ hasta el momento de su análisis. La determinación de coliformes termotolerantes fue definida en el Laboratorio de Microbiología de la Facultad de Zootecnia de la Universidad Nacional del Centro del Perú, según el método del número más probable.

Para la comparación de parámetros físicoquímicos y biológicos determinados se ha utilizado como referencia de comparación los estándares nacionales de calidad ambiental para agua (1).

\section{Identificación y valoración de impactos}

La valoración cualitativa de impactos fue establecida previa identificación de las acciones de las actividades antropogénicas desarrolladas en el río Cunas. Luego se procedió a identificar las acciones de cada una de las actividades que tienen incidencia 
sobre la calidad del agua.

$$
\text { Impacto total }=\mathrm{C} \times(\mathrm{P}+\mathrm{I}+\mathrm{O}+\mathrm{E}+\mathrm{D}+\mathrm{R})
$$

Impacto negativo:

Severo: $\geq(-) 15$

Moderado: (-) 15 $\geq(-) 9$

Compatible: $\leq(-) 9$

Impacto positivo:

Alto: $\geq(+) 15$

Mediano: $(+) 15 \geq(+) 9$

Bajo: $\leq(+) 9$

\section{RESULTADOS}

\section{Calidad de agua del río Cunas}

Los resultados de los parámetros físicoquímicos y biológicos obtenidos sobre la base del protocolo de monitoreo de la calidad sanitaria de los recursos hídricos superficiales de Digesa (2), permitieron determinar la calidad de agua del río Cunas, según el índice NSF. En la Tabla 1, se muestran los valores de los índices NSF obtenidos a partir del cálculo del producto ponderado y el puntaje según el lugar de muestreo del trayecto del río evaluado. La calidad de agua en la estación de Angasmayo fue de 65,83; en la de Antacusi, de 61,08; y en la de La Perla, de 57,18.

Los resultados de la calidad de agua obtenidos en las tres estaciones en estudio fueron interpretados de acuerdo con la escala de clasificación que utiliza el INSF. La calidad del agua del río Cunas obtenida calificó en el rango de 51 a 90, calidad media. Este resultado significa que la mayoría de usos puede darse, aunque las condiciones en pocas veces son cercanas a las naturales.

\section{Identificación de impactos}

La identificación de las acciones posiblemente impactantes en la calidad del agua del río Cunas, según actividad antropogénica, permitió a su vez reconocer los respectivos impactos o efectos que, tanto las actividades como las acciones, dejan en el mencionado río. De acuerdo con la Tabla 2, cada actividad, en función de su naturaleza, genera elementos contaminantes que provocan alteraciones en el estado físico, químico y biológico de las masas de agua del río Cunas. Así, la actividad agrícola, por ejemplo, conlleva al aporte de nitrógeno y fósforo, elementos que provocan eutrofia y la alteración del estado químico en las aguas del río. En general, las consecuencias que ocasionan en el río Cunas las actividades, a través de sus acciones, son eutrofia, alteración del estado químico y turbidez, impactos que

Tabla No 1: Calidad de agua del río Cunas, según el índice NSF.

\begin{tabular}{lclll}
\hline Parámetro & Unidades & Angasmayo & Antacusi & La Perla \\
\hline $\mathrm{pH}$ & Unidad & 8,04 & 8,37 & 8,14 \\
Conductividad & $\mu \mathrm{S} / \mathrm{cm}$ & 430,00 & 450,00 & 450,00 \\
Turbidez & $\mathrm{FTU}$ & 0,00 & 0,00 & 0,00 \\
Oxígeno disuelto & $\mathrm{mg} / \mathrm{L}$ & 7,28 & 6,45 & 6,33 \\
Temperatura & $\mathrm{Celsius}$ & 11,80 & 12,30 & 14,80 \\
Sólidos totales & $\mathrm{mg} / \mathrm{L}$ & 301,00 & 315,00 & 315,00 \\
disueltos & $\mathrm{mg} / \mathrm{L}$ & 0,02 & 0,13 & 0,22 \\
Fosfatos & $\mathrm{mg} / \mathrm{L}$ & 0,00 & 0,00 & 0,00 \\
Nitratos & $\mathrm{NMP} / 100 \mathrm{~mL}$ & 120,00 & 460,00 & 1100,00 \\
Coliformes & & 65,83 & 61,08 & 57,18 \\
Termotolerantes & & & & \\
Índice NFS & & & & \\
\hline
\end{tabular}


Tabla № 2: Acciones posiblemente impactantes en la calidad del agua del río Cunas, según actividad antropogénica.

\begin{tabular}{|c|c|c|}
\hline Actividad & Acciones & Posibles impactos \\
\hline \multirow[t]{2}{*}{ Agrícola } & Aporte de nitrógeno y fósforo & $\begin{array}{l}\text { Eutrofia } \\
\text { Alteración del estado químico }\end{array}$ \\
\hline & Aporte de plaguicidas & Alteración del estado químico \\
\hline Ganadería & Descarga de afluentes líquidos & $\begin{array}{l}\text { Turbidez } \\
\text { Alteración del estado químico }\end{array}$ \\
\hline Piscicultura & Descarga de afluentes líquidos & $\begin{array}{l}\text { Eutrofia } \\
\text { Alteración del estado químico }\end{array}$ \\
\hline \multirow{2}{*}{$\begin{array}{l}\text { Matanza de ganado, } \\
\text { Preparación y conservación } \\
\text { de carne }\end{array}$} & Descarga de afluentes líquidos & $\begin{array}{l}\text { Turbidez } \\
\text { Alteración del estado químico }\end{array}$ \\
\hline & Descarga de residuos sólidos & $\begin{array}{l}\text { Turbidez } \\
\text { Alteración del estado químico }\end{array}$ \\
\hline $\begin{array}{l}\text { Uso doméstico } \\
\text { (asentamientos humanos) }\end{array}$ & Descarga de afluentes líquidos & Alteración del estado químico \\
\hline
\end{tabular}

Tabla Nº 3: Valoración cuantitativa de impactos antropogénicos en la calidad del agua del río Cunas.

\begin{tabular}{|c|c|c|c|c|c|c|}
\hline \multirow{3}{*}{$\begin{array}{c}\text { Característica del } \\
\text { impacto }\end{array}$} & \multicolumn{6}{|c|}{ Medio impactado } \\
\hline & \multicolumn{2}{|c|}{ Angasmayo } & \multicolumn{2}{|c|}{ Antacusi } & \multicolumn{2}{|c|}{ La Perla } \\
\hline & Físico & Biótico & Físico & Biótico & Físico & Biótico \\
\hline Carácter & 1 & 1 & -1 & -1 & -1 & -1 \\
\hline Perturbación & 1 & 1 & 2 & 2 & 3 & 3 \\
\hline Importancia & 3 & 3 & 3 & 3 & 3 & 3 \\
\hline Ocurrencia & 1 & 1 & 2 & 2 & 3 & 3 \\
\hline Extensión & 1 & 1 & 1 & 1 & 1 & 1 \\
\hline Duración & 2 & 2 & 3 & 3 & 3 & 3 \\
\hline Reversibilidad & 1 & 1 & 1 & 2 & 2 & 2 \\
\hline Impacto total & $(-) 10$ & $(-) 10$ & $(-) 13$ & $(-) 14$ & $(-) 16$ & $(-) 16$ \\
\hline
\end{tabular}

degradan la calidad del agua.

\section{Valoración de impactos}

Una vez identificados los impactos que generan las actividades humanas sobre el estado de las masas de agua del río Cunas; medio físico y biótico, se determinó la importancia del efecto a partir del grado de incidencia de la alteración provocada y de una caracterización del mismo, teniendo en cuenta una serie de atributos, tales como: extensión, tipo de efecto, período de manifestación, entre otros.

La estimación de impactos se realizó en base a sus características, tales como carácter, perturbación, importancia, ocurrencia, extensión, duración y reversibilidad en función del índice de calidad de agua determinado. Se asignaron números enteros a cada una de las características, se sumaron por columnas a fin de identificar las acciones más impactantes (valores altos 
negativos) y las menos impactantes (valores bajos negativos) en cada uno de los medios de los sectores evaluados (Tabla 3).

Los resultados exponen que en la estación de muestreo de Angasmayo el impacto total es moderado con tendencia a ser compatible; lo cual dependerá de la época del año. En Antacusi, se obtuvieron impactos moderados tendientes a ser impactos severos; en tanto, en La Perla el impacto total fue severo, tanto en el medio físico como en el biótico.

\section{DISCUSIÓN}

Los resultados obtenidos muestran que en el río Cunas se vienen desarrollando diversas actividades antropogénicas, que generan presiones sobre el entorno humano, natural y socioeconómico. Estas actividades producen una gran variedad de desechos que son liberados principalmente al ambiente acuático. La introducción de un determinado desecho antropogénico puede o no introducir desequilibrios en un ecosistema. Ello dependerá de la cantidad y calidad del desecho introducido (3).

Los resultados obtenidos sobre la calidad del agua del río Cunas para los parámetros físicos-químicos evaluados en las tres estaciones de muestreo (Tabla 1), mostraron que el $\mathrm{pH}$ del agua se encuentra entre los límites de los Estándares de Calidad Ambiental (ECA) nacional para agua, referida a la categorías 3 (riego de vegetales y bebidas de animales) y categoría 4 (conservación del ambiente acuático). Sin embargo, en las tres estaciones de muestreo estos valores estuvieron muy cerca del valor superior de los ECA.

Esta tendencia de los valores de $\mathrm{pH}$ hacia la alcalinidad se debería a las condiciones edáficas del área intervenida y al ingreso de fertilizantes al medio acuático (4). Estos resultados han sido corroborados en otros estudios (5), en los cuales se explican que el $\mathrm{pH}$ del agua varía según las zonas por las que atraviesa la corriente. Si las aguas atraviesan zonas calcáreas el $\mathrm{pH}$ se incrementa, con lo que se tiende a la alcalinidad del medio.

El registro de la conductividad del agua muestra valores que se encuentran dentro de los valores de los ECA para la categoría 3 (Tabla 1); lo cual refleja que el contenido de sales estaría dentro de los valores normales para esta categoría de agua así como para otros usos (6). Igualmente, el registro de este parámetro permitió identificar el ingreso de fertilizantes inorgánicos al medio acuático. Además, al obtenerse in situ a muy bajo costo, es un factor que contribuye a dar sostenibilidad a un programa de seguimiento ambiental (4).

El oxígeno disuelto es sin duda el más importante de los gases disueltos que se encuentra en las aguas naturales (5), porque define en gran parte la biodiversidad y la supervivencia de la comunidad biótica. La medición de oxígeno disuelto obtenida refleja los niveles relativamente buenos de oxígeno en dos de las estaciones evaluadas. Mientras que en la estación 1 (Tabla 1), los niveles de oxígeno disuelto estarían determinando la participación de organismos aerobios en los procesos de degradación, lo que marca la capacidad del agua para llevar a cabo procesos de autopurificación (7).

La temperatura es un factor limitante para la mayoría de los organismos acuáticos y de hecho es una de las constantes que adquiere gran importancia en el desarrollo de los distintos fenómenos que se realizan en el agua, ya que determina la tendencia - evolución de sus propiedades físicas - biológicas (8). Los datos obtenidos en las tres estaciones de muestreo reflejan básicamente una temperatura uniforme, aunque mayor en la estación La Perla, donde el bajo caudal y la pobre cubierta vegetal, debido a la eliminación del bosque ribereño, determinan altas temperaturas ambientales y por ende repercuten en la temperatura del agua, sobre todo en época seca cuando el caudal baja. 
Los datos obtenidos de sólidos totales disueltos y fosfatos no superan los ECA (500 mg/L y $0,5 \mathrm{mg} / \mathrm{L}$, respectivamente), para la conservación del ambiente acuático. Sin embargo, cuando los niveles de concentración de ambos parámetros se incrementan reflejarían problemas de eutrofización.

En relación con la calidad del agua los resultados de los parámetros biológicos en las tres estaciones de muestreo (Tabla 1), mostraron que los niveles de concentración de coliformes termotolerantes se encuentran entre los límites de los estándares nacionales de calidad ambiental para las categorías 3 y 4 . En tanto que para la categoría 1 (poblacional y recreacional) y 3 (riego de vegetales y bebida de animales), el nivel de coliformes termotolerantes supera los ECA, lo cual pone en riesgo la salud de las personas si este recurso es destinado a la producción de agua potable con solamente desinfección.

La evaluación de la calidad del agua mediante el índice NFS ha permitido integrar los parámetros físico-químicos y biológicos, y calificar el tipo de agua en las tres estaciones de muestreo como agua de calidad media, en la que la mayoría de usos pueden darse, tales como producción acuícola, riego de vegetales, bebidas de animales y recreación por contacto secundario. Asimismo, permite observar los impactos de las fuentes de contaminación en variedad de condiciones (4).

Los impactos identificados en el área de intervención corresponden a las fases de operación de las actividades que allí se desarrollan y fueron valorados en función de la Ley General del Ambiente, de la guía para la elaboración de estudios de impacto ambiental (9) y del Decreto Supremo № 002-2008-MINAM. De acuerdo con estas normas, se consideran a las aguas del río Cunas en las categorías 3 y 4 , por tratarse de aguas destinadas para el riego de vegetales y bebidas de animales, así como para la conservación del ambiente acuático, respectivamente, sobre todo en las estaciones de Angasmayo y Antacusi.
En tanto que las aguas del río Cunas en la estación 3 debería ser obviada su uso para riego de vegetales de tallo corto, así como para uso poblacional y de recreación por contacto primario; ya que el nivel de concentración de coliformes termotolerantes superan los ECA para las categorías 1 y 3 .

Los impactos que ocasionan las descargas de afluentes líquidos y la emisión de residuos sólidos sobre la calidad del agua del río Cunas, de acuerdo con el análisis e interpretación de los resultados obtenidos, puede considerarse como un impacto ligeramente moderado en el caso de la estación de muestreo de Angasmayo. Significa que si se suspenden las acciones de las actividades humanas identificadas sobre este factor ambiental, el impacto sería compatible y no precisaría la aplicación de medidas correctivas, habría una recuperación inmediata de los estados químico y ecológico; es decir, las condiciones de calidad del agua estarían cercanas a los niveles naturales (10).

Por ejemplo, en el caso de la estación de Antacusi el impacto también es moderado y la recuperación requiere cierto tiempo, por lo que es aconsejable la aplicación de medidas correctivas. El impacto, en cambio, es severo en la estación La Perla. La magnitud del impacto, en este caso, exige para su recuperación la introducción de medidas correctivas que permitan llevar a niveles aceptables los estados químico y ecológico de las aguas del río Cunas (11, 12). 


\section{REFERENCIAS BIBLIOGRÁFICAS}

1. Ministerio del Ambiente - MINAM. Estándares nacionales de calidad ambiental para aguas. D.S. № 002-2008-MINAM. Norma legal. Lima: MINAM; 2008.

2. Ministerio de Salud - MINSA. Protocolo de monitoreo de la calidad sanitaria de los recursos hídricos superficiales. DIGESA. Área de protección de recursos hídrico. Lima: MINSA; 2007.

3. Arce $M$, Leiva $C$. Determinación de la calidad de agua de los ríos de la ciudad de Loja y diseño de líneas de acción para su recuperación y manejo. [Tesis para optar el título de Ingeniero en Gestión Ambiental]. Loja: Universidad Técnica Particular de Loja; 2009.

4. Pérez C, Rodríguez A. Índice fisicoquímico de la calidad de agua para el manejo de lagunas tropicales de inundación. Rev Biol Trop. 2008; 56 (4): 1905-1918.

5. Martínez L, Pujante M. Estudio de la fauna de invertebrados en el río Cabriel y manantiales asociados en la provincia de Albacete. La Mancha: Centro de Estudios de Castilla; 2008.

6. Carbajal F. Impacto ambiental y social del vertimiento de residuos sólidos y escombros sobre la calidad del río Medellín y de algunos de sus efluentes. Ago Usb. 2009; 9 (1): 225-265.

7. Calla L. Calidad del agua en la cuenca del río Rímac - sector de San Mateo, afectado por las actividades mineras. [Tesis para optar el grado académico de Magíster en Ciencias Ambientales]. Lima: Universidad Mayor de San Marcos; 2010.

8. Bustamante T, Monsalve D, García R. Análisis de la calidad del agua en la cuenca media del río Quindío con base en índices físicos, químicos y biológicos. Rev. Invest. Univ. Quindío. 2008; 1(18): $22-31$.

9. Espinoza G. Gestión y fundamentos de evaluación de impacto ambiental. Banco interamericano de desarrolloBID. Santiago: Centro de estudios para el desarrollo-CED; 2007.

10. Ferrer Y. Evaluación en el tiempo del impacto ambiental con técnicas difusas. Aplicación en la minería de Moa. [Tesis para optar el grado académico de Doctor]. Granada: Universidad de Granada; 2009.

11. Dixon J, Scura L, Carpenter R, Sherman P. Análisis económico de impactos ambientales. Turrialba: Ed. Latinoamericana; 1999.

12. Ministerio de Energía y Minas - MINEM. Guía para la evaluación de impactos en la calidad de agua superficiales por actividades minero metalúrgicas. Lima: Dirección General de Asuntos Ambientales Mineros; 2007. 